\title{
Frobenius Theory for Positive Maps of von Neumann Algebras
}

\author{
Sergio Albeverio and Raphael Hфegh-Krohn \\ Institute of Mathematics, University of Oslo, Blindern, Oslo, Norway
}

\begin{abstract}
Frobenius theory about the cyclic structure of eigenvalues of irreducible non negative matrices is extended to the case of positive linear maps of von Neumann algebras. Semigroups of such maps and ergodic properties are also considered.
\end{abstract}

\section{Introduction}

The spectral theory of positive maps has its origin in the classical work of Perron [1] and Frobenius [2], who considered the case of matrices with positive entries on finite dimensional vector spaces. For a compact exposition of Perron-Frobenius results see [3]. Let us distinguish two types of results in this theory. The first, due to Perron [1], is concerned with the existence and uniqueness of the maximal eigenvalue, the second, due to Frobenius [2], is concerned with the cyclic structure of the spectrum. Frobenius showed more particularly that a non negative irreducible matrix has always a simple eigenvalue $r$ such that all other eigenvalues are contained in a circle of radius $r$ around the origin. If the matrix is normalized such that $r=1$ then the eigenvalues on the unit circle form a finite subgroup of the circle group which maps the system of all eigenvalues into itself.

In this paper we extend Frobenius results to the case of 2-positive maps of von Neumann algebras. Let us first give some references to previous work. As the literature is quite extensive, especially concerning extensions of Perron's results, we shall mainly mention work related to Frobenius results (for additional references see [4]).

Frobenius type of results for compact operators on commutative $\mathrm{C}^{*}$-algebras and ordered vector spaces can be found in Krein and Rutman [5], who also extended Jentsch's work [6] on Perron type of results. For other extensions in the case of ordered vector spaces see e.g. [7]-[9].

Automorphisms of commutative $\mathrm{C}^{*}$-algebras have been studied particularly in connection with ergodic theory, originating from classical work by Koopman [10], Carleman [11] and von Neumann [12], see [13]. Results of Frobenius type for groups of automorphisms in the general case of non commutative $\mathrm{C}^{*}$-algebras have been obtained by St $\phi$ rmer [14]. 
For some particular spectral results which appeared in different contexts see the references in [14] and for recent related results see [15]-[17].

The extension of the entire Perron-Frobenius theory to the case of positive maps on finite-dimensional $\mathrm{C}^{*}$-algebras has been obtained by Evans and HфeghKrohn [4].

We shall now briefly discuss our results.

We consider a von Neumann algebra $M$ and positive linear normalized maps $\Phi$ of $M$ into itself, which are 2-positive and thus satisfy the Kadison-Schwarz inequality $\Phi\left(a^{*} a\right) \geqq \Phi(a)^{*} \Phi(a)$ for any $a \in M$ (see e.g. [18]-[21]).

We recall that a map $\Phi$ is called 2-positive if $\Phi \otimes 1$ is positive on $M \otimes M_{2}$, where 1 is the unit mapping of the space $M_{2}$ of $2 \times 2$ matrices, so that in particular completely positive maps are 2-positive. Such maps have found several applications recently, see e.g. [22]-[26].

Consider now a state invariant under $\Phi$ and extend $\Phi$ to the Hilbert space $\mathscr{H}$ generated by applying $M$ to the cyclic separating vector given by the state. Let $\Phi$ be ergodic, then we show that the set of eigenvalues on the unit circle for $\Phi$ in $M$ and for $\Phi$ in $\mathscr{H}$ is the same, it consists of simple eigenvalues ("roots") $\alpha$ which form a subgroup of the circle group acting by complex multiplication on the spectrum of $\Phi$ as an operator in $\mathscr{H}$. The corresponding eigenvectors give unitary operators $u_{\alpha}$ and the map $\alpha \rightarrow u_{\alpha}$ is a unitary multiplier representation of the group $\Gamma(\Phi)$ of roots. The restriction of $\Phi$ to the subalgebra $M_{\Gamma}$ of $M$ generated by the operators $u_{\alpha}$ is an ergodic automorphism and the restriction of the state to $M_{\Gamma}$ is a trace. We give also more detailed results for the cases where $\Gamma(\Phi)$ is cyclic or finite.

We then extend (Theorem 2.8-2.10) the considerations to the case of semigroups $\Phi_{t}, t \geqq 0$ obtaining Frobenius type of results for their infinitesimal generators.

\section{Dynamical Systems}

Let $M$ be a von Neumann algebra and $\Phi$ a positive linear normalized map of $M$, i.e. $\Phi\left(M^{+}\right) \subseteq M^{+}$, where $M^{+}$is the positive cone in $M$ and $\Phi(1)=1$. We say that $\Phi$ is 2-positive if $\Phi \otimes \mathbb{1}_{2}$ is positive on $M \otimes M_{2}$, where $M_{2}$ is the algebra of $2 \times 2$-matrices and $\mathbb{t}_{2}$ is the identity map of $M_{2}$. It is easily seen that any 2-positive map of $M$ satisfies the Schwarz inequality

$$
\Phi\left(a^{*} a\right) \geqq \Phi(a)^{*} \Phi(a)
$$

for any $a \in M$.

We say that the triplet $(M, \Phi, \xi)$ is a dynamical system if $\Phi$ is a 2-positive map of the von Neumann algebra $M$ and $\xi$ is a faithful normal state on $M$ which is invariant under $\Phi$, i.e. $\xi \circ \Phi=\xi$. Any ${ }^{*}$-automorphism $\theta$ of $M$ is 2 -positive and a dynamical system $(M, \theta, \xi)$, where $\theta$ is a *automorphism is called a closed dynamical system. By the GNS construction we may assume that $\xi$ is a vector state

$$
\xi(a)=(\Omega, a \Omega) .
$$

Let $\mathscr{H}=\bar{M} \Omega$ be the Hilbert space generated by $M$ and the cyclic vector $\Omega$. For $a \in M$ we define 


$$
\Phi a \Omega=\Phi(a) \Omega .
$$

By (2.1) we then have

$$
\|\Phi a \Omega\|^{2}=\xi\left(\Phi(a)^{*} \Phi(a)\right) \leqq \xi\left(\Phi\left(a^{*} a\right)\right)=\xi\left(a^{*} a\right)=\|a\|^{2} .
$$

Hence $\Phi$ with dense domain $M \Omega$ is a contraction on $\mathscr{H}$ and thus extends uniquely to a contraction on $\mathscr{H}$.

Let $a \in M$ and $b^{\prime} \in M^{\prime}$, where $M^{\prime}$ is the commutant of $M$. Let $\xi$ on $B(\mathscr{H})$ be given by (2.2), then $b^{\prime} \in\left(M^{\prime}\right)^{+}$if and only if $\xi\left(a b^{\prime}\right) \geqq 0$ for all $a \in M^{+}$, since $\Omega$ is a cyclic and separating vector for $M$. Now if $b^{\prime} \in\left(M^{\prime}\right)^{+}$then for $a \in M^{+}$we have

$$
\xi\left(\Phi(a) b^{\prime}\right) \leqq\left\|b^{\prime}\right\| \xi(\Phi(a))=\left\|b^{\prime}\right\| \xi(a) .
$$

Hence if $b^{\prime} \in\left(M^{\prime}\right)^{+}$is normalized such that $\xi\left(b^{\prime}\right)=1$, then $\xi\left(\Phi(a) b^{\prime}\right)$ is a state on $M$ which is majorized by the state $\xi$. It is well known that such a state is of the form

$$
\xi\left(\Phi(a) b^{\prime}\right)=\xi\left(a \Phi^{\prime}\left(b^{\prime}\right)\right)
$$

where $\Phi^{\prime}\left(b^{\prime}\right)$ is some positive operator affiliated with $M^{\prime}$. The correspondence $b^{\prime} \rightarrow \Phi^{\prime}\left(b^{\prime}\right)$ is obviously linear and monotone and $\xi \circ \Phi=\xi$ implies that $\Phi^{\prime}(1)=1$. By the monotonicity we then get for $b^{\prime} \in\left(M^{\prime}\right)^{+}$that $\Phi^{\prime}\left(b^{\prime}\right) \leqq\left\|b^{\prime}\right\|$. Hence $\Phi^{\prime}$ is a positive map of $M^{\prime}$. Since $\left(M \otimes M_{2}\right)^{\prime} \simeq M^{\prime} \otimes M_{2}$ and if $\alpha \in M \otimes M_{2}$ and $\beta^{\prime} \in\left(M \otimes M_{2}\right)^{\prime}$ then

$$
\xi \otimes \tau_{2}\left(\Phi \otimes \mathbb{1}_{2}(\alpha) \beta^{\prime}\right)=\xi \otimes \tau_{2}\left(\alpha\left(\Phi^{\prime} \otimes \mathbb{1}_{2}\right)(\beta)\right),
$$

where $\tau_{2}$ is the normalized trace on $M_{2}$, we see that $\left(\Phi \otimes \mathbb{1}_{2}\right)^{\prime}=\Phi^{\prime} \otimes \mathbb{1}_{2}$. This together with the above argument gives us that $\Phi^{\prime}$ is a 2-positive map of $M^{\prime}$.

Moreover $\Phi(1)=1$ implies that $\xi \circ \Phi^{\prime}=\xi$ and therefore $\left(M^{\prime}, \Phi^{\prime}, \xi\right)$ is again a dynamical system. We thus have the following theorem

Theorem 2.1. Let $(M, \Phi, \xi)$ be a dynamical system. If $\mathscr{H}=\bar{M} \Omega$ is the Hilbert space generated by $M$ and $\Omega$, where $\Omega$ is the vector given by the state $\xi$, then for $a \in M$ and $b^{\prime} \in M^{\prime}$ the equation

$$
\xi\left(\Phi(a) b^{\prime}\right)=\xi\left(a \Phi^{\prime}\left(b^{\prime}\right)\right)
$$

defines uniquely a 2-positive map of the commutant $M^{\prime}$ such that $\left(M^{\prime}, \Phi^{\prime}, \xi\right)$ is a dynamical system. Moreover $\Phi a \Omega=\Phi(a) \Omega$ defines a contraction on $M \Omega$ which extends to a contraction on $\mathscr{H}$.

We shall call $\left(M^{\prime}, \Phi^{\prime}, \xi\right)$ the dual dynamical system. We say that a dynamical system $(M, \Phi, \xi)$ is ergodic if $\xi$ is the only normal state on $M^{\prime}$ which is invariant under $\Phi^{\prime}$. The following lemma follows from the fact that if $a \in M^{+}, \xi(a)=1$, then $\xi\left(a b^{\prime}\right)$ is a normal state on $M^{\prime}$.

Lemma 2.1. If $(M, \Phi, \xi)$ is an ergodic dynamical system then the only elements in $M$ invariant under $\Phi$ are those proportional to the identity.

From (2.1) we get with $a \in M$ and $b^{\prime} \in\left(M^{\prime}\right)^{+}$that

$$
\text { or } \begin{aligned}
\xi\left(b^{\prime} \Phi\left(a^{*} a\right)\right) & \geqq \xi\left(\Phi(a)^{*} b^{\prime} \Phi(a)\right) \\
& \xi\left(a^{*} \Phi^{\prime}\left(b^{\prime}\right) a\right) \geqq \xi\left(\Phi(a)^{*} b^{\prime} \Phi(a)\right)
\end{aligned}
$$


which gives

$$
\left(a \Omega, \Phi^{\prime}\left(b^{\prime}\right) a \Omega\right) \geqq\left(\Phi a \Omega, b^{\prime} \Phi a \Omega\right) .
$$

Since $M \Omega$ is dense in $\mathscr{H}$ we have for arbitrary $x \in \mathscr{H}$ that for $b^{\prime} \in\left(M^{\prime}\right)^{+}$

$$
\left(x, \Phi^{\prime}\left(b^{\prime}\right) x\right) \geqq\left(\Phi x, b^{\prime} \Phi x\right) .
$$

Since $\Phi$ is a contraction on $\mathscr{H}$, the spectrum of $\Phi$ is contained inside the unit circle. If $x_{\alpha}$ is an eigenvector of $\Phi$ corresponding to an eigenvalue $\alpha$ on the unit circle i.e.

$$
\Phi x_{\alpha}=\alpha x_{\alpha}, \quad|\alpha|=1
$$

we say that $x_{\alpha}$ is a root vector for $\Phi$ corresponding to the root $\alpha$. The set of roots $\Gamma(\Phi)$ of $\Phi$ consists then of the eigenvalues for $\Phi$ on the unit circle. Let now $x_{\alpha}$ be a normalized root vector, by (2.11) we then have

$$
\left(x_{\alpha}, \Phi^{\prime}\left(b^{\prime}\right) x_{\alpha}\right) \geqq\left(x_{\alpha}, b^{\prime} x_{\alpha}\right)
$$

for any $b^{\prime} \in\left(M^{\prime}\right)^{+}$. Both sides of the inequality (2.13) define normalized states on $M^{\prime}$ and since one dominates the other they must be equal, hence

$$
\left(x_{\alpha}, \Phi^{\prime}\left(b^{\prime}\right) x_{\alpha}\right)=\left(x_{\alpha}, b^{\prime} x_{\alpha}\right)
$$

for arbitrary $b^{\prime} \in M^{\prime}$. Thus the vector state $b^{\prime} \rightarrow\left(x_{\alpha}, b^{\prime} x_{\alpha}\right)$ corresponding to a root vector $x_{\alpha}$ is an invariant state for $\Phi^{\prime}$.

Let us now assume that the dynamical system $(M, \Phi, \xi)$ is ergodic, then we have that

$$
\left(x_{\alpha}, b^{\prime} x_{\alpha}\right)=\xi\left(b^{\prime}\right)
$$

for any $b^{\prime} \in M^{\prime}$. Set now $b^{\prime}=c^{\prime *} c^{\prime}$, then (2.15) gives us

$$
\left\|c^{\prime} x_{\alpha}\right\|=\left\|c^{\prime} \Omega\right\| \text {. }
$$

Let us now define the densely defined operator $\hat{x}_{\alpha}$ by

$$
\hat{x}_{\alpha} c^{\prime} \Omega=c^{\prime} x_{\alpha}
$$

for $c^{\prime} \in M^{\prime}$ i.e. $D\left(\hat{x}_{\alpha}\right)=M^{\prime} \Omega$. From (2.16) we then have

$$
\left\|\hat{x}_{\alpha} c^{\prime} \Omega\right\|=\left\|c^{\prime} \Omega\right\|
$$

hence $\hat{x}_{\alpha}$ is an isometry

$$
\hat{x}_{\alpha}^{*} \hat{x}_{\alpha}=1 \text {. }
$$

From the definition (2.17) we have that

$$
\hat{x}_{\alpha} b^{\prime}=b^{\prime} \hat{x}_{\alpha}
$$

for arbitary $b^{\prime} \in M^{\prime}$. Thus $\hat{x}_{\alpha} \in M$ and by (2.12) we have

$$
\Phi\left(\hat{x}_{\alpha}\right)=\alpha \hat{x}_{\alpha} .
$$

Since $\Phi$ is a positive map of $M$ we have that

$$
\Phi\left(a^{*}\right)=\Phi(a)^{*},
$$


which gives us that

$$
\Phi\left(\hat{x}_{\alpha}^{*}\right)=\bar{\alpha} \hat{x}_{\alpha}^{*} .
$$

Thus if $\alpha$ is a root so is $\bar{\alpha}$ and the corresponding root vector is $\hat{x}_{\alpha}^{*} \Omega$. Now (2.19) with $\hat{x}_{\alpha}^{*}$ replacing $\hat{x}_{\alpha}$ gives

$$
\hat{x}_{\alpha} \cdot \hat{x}_{\alpha}^{*}=1 \text {, }
$$

so that $\hat{x}_{\alpha}$ is unitary.

Let us now consider the quadratic form defined on $M$ by

$$
\mu_{b^{\prime}}(a, a)=\xi\left(b^{\prime}\left(\Phi\left(a^{*} a\right)-\Phi(a)^{*} \Phi(a)\right)\right) .
$$

Then by (2.1) we have for $b^{\prime} \in\left(M^{\prime}\right)^{+}$that $\mu_{b^{\prime}}(a, a)$ is a positive semi-definite form. From (2.19) and (2.21) we have that

$$
\mu_{b^{\prime}}\left(\hat{x}_{\alpha}, \hat{x}_{\alpha}\right)=0 \text {. }
$$

Thus by Schwarz inequality we have

$$
\mu_{b^{\prime}}\left(\hat{x}_{\alpha}, a\right)=0
$$

for arbitrary $b^{\prime} \in\left(M^{\prime}\right)^{+}$and $a \in M$. Since $\Omega$ is separating and cyclic also for $M^{\prime}$ we therefore have

$$
\left.\Phi\left(\hat{x}_{\alpha}^{*} a\right)=\bar{\alpha} \hat{x}_{\alpha}^{*} \Phi(a)\right) .
$$

which again implies that

$$
\Phi\left(\hat{x}_{\alpha} a\right)=\alpha \hat{x}_{\alpha} \Phi(a),
$$

$\hat{x}_{\alpha} \Omega$ being again a root vector. Since $M \Omega$ is dense in $\mathscr{H}$ we get from (2.28) that

$$
\hat{x}_{\alpha}^{*} \Phi \hat{x}_{\alpha}=\alpha \Phi \text {. }
$$

Still under the assumption that $(M, \Phi, \xi)$ is an ergodic dynamical system we get from (2.29) that if $\alpha \in \Gamma(\Phi)$ then the operators $\Phi$ and $\alpha \Phi$ are unitary equivalent. Especially we get that if $\alpha$ and $\beta$ are in $\Gamma(\Phi)$ then $\alpha \cdot \beta$ is in $\Gamma(\Phi)$, and we already had that if $\alpha \in \Gamma(\Phi)$ then $\bar{\alpha} \in \Gamma(\Phi)$. Hence $\Gamma(\Phi)$ is a subgroup of the circle group. Let $\alpha \in \Gamma(\Phi)$ and let $u_{\alpha}$ be the corresponding unitary root operator in $M$. From (2.28) we get that if $\alpha$ and $\beta$ are in $\Gamma(\Phi)$ then

$$
\Phi\left(u_{\alpha} u_{\beta}\right)=\alpha \beta u_{\alpha} u_{\beta}
$$

so that $u_{\alpha} u_{\beta}$ is a root operator corresponding to the root $\alpha \beta$. Let $u_{\alpha}$ and $v_{\alpha}$ be two unitary root operators corresponding to the same root $\alpha$. By (2.27) we then have

$$
\Phi\left(u_{\alpha}^{*} v_{\alpha}\right)=u_{\alpha}^{*} v_{\alpha}
$$

which by Lemma 2.1 gives us the following

$$
u_{\alpha}^{*} v_{\alpha}=c 1,|c|=1 \text {, }
$$

so that $v_{\alpha}=c u_{\alpha}$. Hence we have proved that any root $\alpha$ is a simple eigenvalue for $\Phi$. Especially we get that for $\alpha$ and $\beta$ in $\Gamma(\Phi)$

$$
u_{\alpha} u_{\beta}=\gamma(\alpha, \beta) u_{\alpha \beta}
$$


with

$$
|\gamma(\alpha, \beta)|=1 \text {. }
$$

So that

$$
\alpha \rightarrow u_{\alpha}
$$

is a unitary multiplier representation of the group $\Gamma(\Phi)$, with multiplier $\gamma(\alpha, \beta)$. If $\Gamma(\Phi)$ is cyclic, i.e. has a simple generator, then the multiplier $\gamma(\alpha, \beta)$ is necessarily trivial, hence $\alpha \rightarrow u_{\alpha}$ is a unitary representation of the abelian group $\Gamma(\Phi)$. Therefore in this case the algebra generated by the root operators is abelian and

$$
u_{\alpha}^{*}=u_{\alpha} .
$$

Note that one may choose (2.36) also in the general case.

We have now proved the following theorem.

Theorem 2.2. Let $(M, \Phi, \xi)$ be an ergodic dynamical system, where $\xi$ is a cyclic separating vector state for $M$ invariant under $\Phi$. Let $\mathscr{H}$ be the corresponding Hilbert space. Then the discrete eigenvalues on the unit circle for $\Phi$ as an operator in $\mathscr{H}$ coincide with the discrete eigenvalues on the unit circle for $\Phi$ in $M$. Let $\Gamma(\Phi)$ be the set of all roots of $\Phi$, i.e. the discrete eigenvalues on the unit circle. $\Gamma(\Phi)$ is a subgroup of the circle group which acts by complex multiplication on the spectrum $\operatorname{Sp}(\Phi)$ of $\Phi$ in $\mathscr{H}$. If $\alpha \in \Gamma(\Phi)$ then $\alpha$ is a simple eigenvalue of $\Phi$ and the corresponding root operator $u_{\alpha}$ in $M$ is proportional to a unitary operator in $M$ and $x_{\alpha}=u_{\alpha} \Omega$ is the corresponding root vector in $\mathscr{H}$, where $\Omega$ is the vector corresponding to the vector state $\xi$. The invariance of $\operatorname{Sp}(\Phi)$ under multiplication by the root $\alpha$ is given by the unitary equivalence

$$
u_{\alpha}^{*} \Phi u_{\alpha}=\alpha \Phi,
$$

if the root operator $u_{\alpha}$ is normalized so that it is unitary. If $\alpha$ and $\beta$ are in $\Gamma(\Phi)$ with root operators $u_{\alpha}$ and $u_{\beta}$, then $u_{\alpha} u_{\beta}$ is a root operator for the root $\alpha \beta$ and $u_{\alpha}^{*}$ is a root operator for $\bar{\alpha}$. Hence if we select for each $\alpha \in \Gamma(\Phi)$ a unitary operator $u_{\alpha}$ then $u_{\alpha} u_{\beta}=$ $\gamma(\alpha, \beta) u_{\alpha \beta}$, where $\gamma(\alpha, \beta)$ is a multiplier for the group $\Gamma(\Phi)$ and $\alpha \rightarrow u_{\alpha}$ is a unitary multiplier representation of the group $\Gamma(\Phi)$ with multiplier $\gamma(\alpha, \beta)$. If $\Gamma(\Phi)$ is cyclic, i.e. has a single generator, then $\alpha \rightarrow u_{\alpha}$ is a unitary representation of the abelian group $\Gamma(\Phi)$ and therefore the algebra generated by the root operators is abelian.

Remark. Results of this type were proven by Frobenius [2] for commutative, finite-dimensional von Neumann algebras. For the commutative infinite dimensional case with $\Phi$ compact, results were given by Krein and Rutman [5] and for the commutative infinite dimensional case with $\Phi$ an automorphism by Koopman [10] and von Neumann [12]. In the infinite dimensional non-commutative case with $\Phi$ an automorphism results of this type were obtained by St $\phi$ rmer [14] and in the finite dimensional non-commutative case with general $\Phi$ by Evans and Høegh-Krohn [4].

If $\Phi$ is compact in $\mathscr{H}, \Gamma(\Phi)$ must be a finite subgroup of the unit circle and since any such group has the form

$$
\Gamma_{m}=\left\{e^{2 \pi i k / m}, k=0,1, \ldots, m-1\right\}
$$


we have that $\Gamma(\Phi)=\Gamma_{m}$ where $m=|\Gamma(\Phi)|$ is the order of $\Gamma(\Phi)$. We shall say that $\Phi$ is primitive if $|\Gamma(\Phi)|=1$ i.e. $\Gamma(\Phi)=\{1\}$ and imprimitive if not, and following Frobenius we call $|\Gamma(\Phi)|$ the imprimitivity of $\Phi$. Especially we have that if $\Phi$ is compact in $\mathscr{H}$ then it has finite imprimitivity. If $\Phi$ is of trace class in $\mathscr{H}$ then the Fredholm determinant $|1-z \Phi|$ of $1-z \Phi$ exists and defines an entire function

$$
f_{\Phi}(z)=|1-z \Phi|
$$

such that $f_{\Phi}\left(z_{0}\right)=0$ if and only if $z_{0}^{-1}$ is an eigenvalue for $\Phi$. Especially we get that the set of zeros of $f$ on the unit circle is $\Gamma(\Phi)$. Recalling now that for $\alpha \in \Gamma(\Phi)$

$$
u_{\alpha}^{*} \Phi u_{\alpha}=\alpha \Phi
$$

by the unitary equivalence of $\alpha \Phi$ and $\Phi$, we get then

$$
f_{\Phi}(\alpha z)=f_{\Phi}(z)
$$

because the Fredholm determinant is a unitary invariant. Since $\alpha$ in (2.40) is any $m$ th root of the unit and $f$ is entire, we have that there exists an entire function $g(z)$ such that $f_{\Phi}(z)=g\left(z^{m}\right)$. Let us also remark that since $\Gamma(\Phi)=\Gamma_{m}$ is cyclic, we have that the algebra generated by the root operators is commutative. Let now $\gamma=e^{2 \pi i / m}$ and $u^{\prime}$ be a root operator corresponding to $\gamma$ then $u^{\prime m}=\bar{c} .1$ where $|\bar{c}|=1$. Let now $u=c^{1 / m} u^{\prime}$ then $u^{m}=1$. Since $u$ is unitary and $u^{m}=1$ we have the spectral decomposition

$$
u=\sum_{k=0}^{m-1} \gamma^{k} P_{k}
$$

where $P_{k}$ are the spectral projections for $u$.

Since $\Phi(u)=\gamma u$ we see that

$$
\Phi\left(P_{k}\right)=P_{k-1} \text { and } \Phi\left(P_{0}\right)=P_{m-1}, k=1, \ldots, m-1 .
$$

Especially we have that

$$
\Phi^{m}\left(P_{k}\right)=P_{k}
$$

so that $\Phi^{m}$ is not ergodic. It is easy to see that the restriction of $\Phi^{m}$ to the algebra $M_{k}=P_{k} M P_{k}$ is ergodic and in fact primitive. These results depend obviously only on the fact that $\Gamma(\Phi)$ is of finite order. We have thus the following theorem

Theorem 2.3. Let $(M, \Phi, \xi)$ be as in Theorem 2.2. Then if $\Phi$ has finite imprimitivity we have $\Gamma(\Phi)=\Gamma_{m}$, where $\Gamma_{m}$ is the group of $m$-th roots of the unit. Let $\gamma=e^{2 \pi i / m}$ then a root operator $u$ corresponding to $\bar{\gamma}$ may be normalized so that $u^{m}=1$. For this $u$ we have that $u=\sum_{k=0}^{m-1} \gamma^{k} P_{k}$ is the spectral resolution of the unitary operator $u$. Hence $\left\{P_{k}\right\}$ is a resolution of the identity in $M$ and the algebra generated by the root operators is the abelian algebra generated by $\left\{P_{k}\right\}$. Moreover $\Phi\left(P_{k}\right)=P_{k-1}$ and $\Phi\left(P_{0}\right)=P_{m-1}$. Especially $\Phi^{m}\left(P_{k}\right)=P_{k}$, so that $\Phi^{m}$ is not ergodic. However the restriction of $\Phi^{m}$ to the algebra $M_{k}=P_{k} M P_{k}$ is ergodic and primitive. In fact $|\Gamma(\Phi)|=m$ only if $\Phi^{m}$ is not ergodic. If $\Phi$ is compact, then $\Phi$ has finite imprimitivity. If in addition $\Phi$ is of 
trace class in $B(\mathscr{H})$, then there is an entire function $g(z)$ such that

$$
|1-z \Phi|=g\left(z^{m}\right)
$$

where $|1-z \Phi|$ is the Fredholm determinant of $\Phi$.

Let now $\Gamma(\Phi)$ be cyclic but not finite. Then for any root $\gamma \in \Gamma(\Phi)$ we have that $\gamma / 2 \pi$ is irrational. If $\gamma$ generates $\Gamma(\Phi)$ then

$$
\Gamma(\Phi)=\left\{\gamma^{n} ; n=0, \pm 1, \ldots\right\} .
$$

Let now $u$ be a root operator corresponding to $\bar{\gamma}$, normalized so that $u$ is unitary. A root operator corresponding to $\gamma^{n}$ is then given by $u^{-n}$. Let $v$ be the spectral measure on the unit circle for the unitary operator $u$. Since obviously $\Phi$ restricted to the subalgebra generated by $u$ is an automorphism, we have that $\Phi$ induces a transformation of the spectrum of $u$, and since $\Phi\left(u^{n}\right)=\gamma^{n} u^{n}$ it follows that this transformation coincides with the restriction to the spectrum of $u$ of the transformation $z \rightarrow \gamma z$. Hence if

$$
u=\int_{|z|=1} z d E_{z}
$$

is the spectral resolution of $u$, we must have that

$$
\Phi\left(E_{z}\right)=E_{\gamma z}
$$

for $v$-almost all $z$ in the unit circle. Since there are no other root operators than the $u^{n}, n=0, \pm 1, \ldots$, it follows that $v$ is ergodic with respect to the transformation $z \rightarrow \gamma z$ of the unit circle. That $v$ is invariant under this transformation follows from $\xi=\xi \circ \Phi$ and $\Phi\left(u^{n}\right)=\gamma^{n} u^{n}$ for all $n \in \mathbb{Z}$. Hence we have proved the following theorem.

Theorem 2.4. Let $(M, \Phi, \xi)$ be an ergodic dynamical system, such that $\Gamma(\Phi)$ is cyclic but not finite. Then for any $\gamma \in \Gamma(\Phi)$ we have that $\gamma / 2 \pi$ is irrational. Let $\gamma$ generate $\Gamma(\Phi)$, i.e. $\Gamma(\Phi)=\left\{\gamma^{n} ; n=0, \pm 1, \pm 2, \ldots\right\}$. Let $u$ be the root operator corresponding to $\bar{\gamma}$ normalized so that $u$ is unitary.

Let $v$ be the spectral measure on the unit circle for the unitary operator $u$ corresponding to the state $\xi$, i.e. $\xi\left(u^{n}\right)=\int_{|z|=1} z^{n} d v(z)$, and let $u=\int_{|z|=1} z d E_{z}$ be the spectral resolution of $u$. Then the projection valued measure $d E_{z}$ is absolutely continuous with respect to $v . v$ is an invariant ergodic measure with respect to the transformation $z=\gamma z$ of the unit circle and

$$
\Phi\left(E_{z}\right)=E_{\gamma z}
$$

for $v$-almost all $z$ on the unit circle.

Let now $\alpha$ and $\beta$ be two roots of the ergodic dynamical system $(M, \Phi, \xi)$ with corresponding root operators $u_{\alpha}$ and $u_{\beta}$. Since $u_{\alpha} \cdot u_{\beta}$ then is a root operator for the root $\alpha \cdot \beta$ we have

$$
\Phi\left(u_{\alpha} u_{\beta}\right)=\alpha \beta u_{\alpha} u_{\beta}=\Phi\left(u_{\alpha}\right) \cdot \Phi\left(u_{\beta}\right) .
$$

Hence if $M_{\Gamma}$ is the strongly closed subalgebra of $M$ generated by the root operators $u_{\alpha}, \alpha \in \Gamma \equiv \Gamma(\Phi)$, then $\Phi$ maps $M_{\Gamma}$ into $M_{\Gamma}$ and restriction of $\Phi$ to $M_{\Gamma}$ is an auto- 
morphism. Let $\mathscr{H}_{\Gamma}=\overline{M_{\Gamma} \Omega}$ then $\mathscr{H}_{\Gamma}$ is a $\Phi$ invariant subspace of $\mathscr{H}$ and the restriction of $\Phi$ to $\mathscr{H}_{\Gamma}$ is obviously unitary with discrete spectrum equal to $\Gamma$, and $u_{\alpha} \Omega, \alpha \in \Gamma$ is a complete set of orthogonal eigenvectors for $\Phi$ in $\mathscr{H}_{\Gamma}$. Hence $\Omega$ is the only invariant eigenvector. Since $\Phi$ is ergodic on $M$ the restriction of $\Phi$ to $M_{\Gamma}$ is ergodic. From the orthogonality of $\Omega$ and $u_{\alpha} \Omega$ for $\alpha \neq 1$ we have that $\xi\left(u_{\alpha}\right)=0$ for $\alpha \neq 1$. But then $\xi\left(u_{\alpha} u_{\beta}\right)=\xi\left(u_{\beta} u_{\alpha}\right)=0$ for $\alpha \neq \bar{\beta}$ and if $\beta=\bar{\alpha}$ then $u_{\beta}=c u_{\alpha}^{*}$, where $c$ is an element in the unit circle, and since $u_{\alpha}$ is unitary we have that if $\beta=\bar{\alpha}$ then $u_{\alpha} u_{\beta}=u_{\beta} u_{\alpha}$ so that $\xi\left(u_{\alpha} u_{\beta}\right)=\xi\left(u_{\beta} u_{\alpha}\right)$ in any case. This shows that for $a$ and $b$ in $M_{\Gamma}$ then $\xi(a b)=\xi(b a)$ i.e. the restriction of $\xi$ to $M_{\Gamma}$ is a trace. (That the restriction of an ergodic state to the root algebra $M_{\Gamma}$ is a trace was observed by Stormer [14] in the case where $\Phi$ is an automorphism.) We have thus proven the following theorem.

Theorem 2.5. Let $(M, \Phi, \xi)$ be an ergodic dynamical system with root system $\Gamma$. Let $M_{\Gamma}$ be the root algebra, i.e. the strongly closed subalgebra of $M$ generated by the root operators and let $\Phi_{\Gamma}$ be the restriction of $\Phi$ to $M_{\Gamma}$. Then $\Phi_{\Gamma}$ is an automorphism of $M_{\Gamma}$ and $\left(M_{\Gamma}, \Phi_{\Gamma}, \xi_{\Gamma}\right)$, where $\xi_{\Gamma}$ is the restriction of $\xi$ to $M_{\Gamma}$, is an ergodic dynamical system. Moreover $\xi_{\Gamma}$ is a trace on $M_{\Gamma}$.

One could now ask if it is so that $M_{\Gamma}$ is always commutative for an ergodic dynamical system. The following example shows that this is not the case.

Example 2.6. Let $\mathscr{H}=L_{2}(\mathbb{R})$ and $\left.\operatorname{set}(V(x) f) y\right)=f(y-x)$ and $\left.(U(x) f) y\right)=e^{i x y} f(y)$. Then $V$ and $U$ are both strongly continuous unitary representations of the abelian group $\mathbb{R}$ on $L_{2}(\mathbb{R})$. Moreover

$$
U(x) V(y)=e^{i x y} V(y) U(x) .
$$

Let $\lambda \geqq 0$ and $n_{1}, n_{2}$ in $\mathbb{Z}$, then

$$
U\left(\lambda n_{1}\right) V\left(\lambda n_{2}\right)=e^{i \lambda^{2} n_{1} n_{2}} V\left(\lambda n_{1}\right) U\left(\lambda n_{2}\right) .
$$

Let $u_{n} \equiv U\left(\lambda n_{1}\right) V\left(\lambda n_{2}\right)$ for $n=\left(n_{1}, n_{2}\right) \in \mathbb{Z}^{2}$. Then $n \rightarrow u_{n}$ is a unitary multiplier representation of $\mathbb{Z}^{2}$. Let $C$ be the $C^{*}$-algebra spanned by $u_{n}$ and set $\tau\left(u_{n}\right)=\delta_{0 n}$. It is easy to see that $\tau$ defines a faithful trace on $C$. In fact let $a=\sum \alpha_{n} u_{n}$, then we have $\tau\left(a^{*} a\right)=\sum \bar{\alpha}_{n} \alpha_{m} \tau\left(u_{n}^{*} u_{m}\right)=\sum\left|\alpha_{n}\right|^{2}$, which shows that $\tau$ is faithful. Moreover we have $\tau\left(u_{n}^{*} u_{m}\right)=0=\tau\left(u_{m} u_{n}^{*}\right)$ for $n \neq m$ and $\tau\left(u_{n}^{*} u_{n}\right)=1=\tau\left(u_{n} u_{n}^{*}\right)$, which shows that $\tau$ is a trace on $C$. Let now $M$ be the von Neumann algebra given by the representation of $C$ induced by the trace $\tau$. Then $M$ is non commutative if and only if $\lambda^{2}$ is not an integral multiple of $2 \pi$. Let $\alpha$ and $\beta$ be two real numbers and set $W=U(\alpha) V(\beta)$. Then

$$
W^{*} U(\lambda n) W=V^{*}(\beta) U(\lambda n) V(\beta)=e^{i \lambda \beta n} U(\lambda n)
$$

and

$$
W^{*} V(\lambda n) W=V^{*}(\beta) U^{*}(\alpha) V(\lambda n) U(\alpha) V(\beta)=e^{-i \lambda \alpha n} V(\lambda n) .
$$

Set now for $a \in M, \theta(a)=W^{*} a W$, then $\theta$ is an automorphism of $M$ and $(M, \theta, \xi)$ is a dynamical system. Moreover it follows from the above equations that if $\alpha, \beta$ and $2 \pi / \lambda$ are independent over $\mathbb{Z}$ (the ring of integers) then $(M, \theta, \xi)$ is an ergodic dynamical system. We have from the equation above that the root system 
$\Gamma=\Gamma(\theta)$ is given by

$$
\Gamma=\left\{e^{i \lambda(\alpha m+\beta n)} ;(m, n) \in \mathbb{Z} \times \mathbb{Z}\right\}
$$

and a root operator corresponding to $e^{i \lambda(\alpha m+\beta n)}$ is given by $U(\lambda m) V(-\lambda n) . M$ is noncommutative if $\lambda^{2}$ is not an integral multiple of $2 \pi$, and $M=M_{\Gamma}$.

We shall now consider the case of semigroups of positive maps, instead of the iterates of a single positive map $\Phi$.

Let $M$ be a von Neumann algebra and $\Phi_{t}, t \geqq 0$ a semigroup of positive normalized maps of $M$ i.e. $\Phi_{0}=1, \Phi_{t} \circ \Phi_{s}=\Phi_{t+s}, \Phi_{t}\left(M^{+}\right) \subseteq M^{+}$and $\Phi_{t}(1)=1$ such that the $\Phi_{t}$ are 2-positive, hence satisfy the Schwarz inequality

$$
\Phi_{t}\left(a^{*} a\right) \geqq \Phi_{t}(a)^{*} \Phi_{t}(a)
$$

for any $a \in M$ and all $t$. Moreover if $\xi$ is a cyclic and separating normal state on $M$ such that $\xi\left(a \Phi_{t}(b)\right)$ is measurable as a function of $t$, and $\xi$ is invariant under $\Phi_{t}$ i.e. $\xi \circ \Phi_{t}=\xi$, we say that $\left(M, \Phi_{t}, \xi\right)$ is a dynamical system with continuous time or a dynamical flow. We say that the dynamical flow is ergodic iff $\xi$ is the only invariant normal state for the dual flow $\left(M^{\prime}, \Phi_{t}^{\prime}, \xi\right)$. As for the discrete dynamical systems $(M, \Phi, \xi)$ considered before, (2.48) implies that $\Phi_{t}$ extends to a measurable, hence strongly continuous, contraction semigroup on $\mathscr{H}$, where $\mathscr{H}$ is the Hilbert space obtained by the GNS construction from the state $\xi$. We denote the continuous extension to $\mathscr{H}$ also by $\Phi_{t}$, and we let $i A$ be the infinitesimal generator of $\Phi_{t}$ in $\mathscr{H}$ i.e.

$$
\Phi_{t}=e^{i t A} \quad t \geqq 0 .
$$

Since $\Phi_{t}$ is a contraction, we have that $i\left(A-A^{*}\right) \geqq 0$ so that the spectrum of $A$ is confined to the closed upper half plane. Let $\Gamma$ be the discrete part of the spectrum of $A$ on the real line. Then of course for any $t \geqq 0$ we have that $e^{i t \Gamma}$ is the discrete spectrum of $\Phi_{t}$ on the unit circle. Let now $\alpha \in \Gamma$ and $x_{\alpha}$ be a corresponding normalized eigenvector. As in the proof of Theorem 2.2 we get that $x_{\alpha}=u_{\alpha} \Omega$ with $u_{\alpha}$ unitary in $M$. In this way we prove the following theorem.

Theorem 2.7. Let $\left(M, \Phi_{t}, \xi\right)$ be an ergodic dynamical flow. Then the discrete eigenvalues on the real line for the infinitesimal generator of $\Phi_{t}$ in $\mathscr{H}$ coincide with the discrete eigenvalues on the real line for the infinitesimal generator of $\Phi_{t}$ in $M$. Let the set of these discrete eigenvalues on the real line be denoted by $\Gamma$, the root system of the flow, then $\Gamma$ is a subgroup of the additive group of the real line. Moreover the spectrum of the semigroup $\Phi_{t}$ in $\mathscr{H}$ is invariant under this additive group $\Gamma$. Moreover, for any $\alpha \in \Gamma, \alpha$ is a simple eigenvalue of the semigroup $\Phi_{t}$ and a corresponding root operator $u_{\alpha} \in M$ is proportional to a unitary operator in $M$. The invariance of the spectrum of the semigroup $\Phi_{t}$ is given by the unitary equivalence

$$
u_{\alpha}^{*} \Phi_{t} u_{\alpha}=e^{2 \pi i \alpha t} \Phi_{t}
$$

where $u_{\alpha}$ is a normalized root operator corresponding to $\alpha \in \Gamma$. If $\alpha$ and $\beta$ are in $\Gamma$ with root operators $u_{\alpha}$ and $u_{\beta}$ then $u_{\alpha} u_{\beta}$ is a root operator for $\alpha+\beta$ and $u_{\alpha}^{*}$ is a root operator for $-\alpha$. Hence if we select for each $\alpha \in \Gamma$ a unitary root operator $u_{\alpha}$ then $u_{\alpha} u_{\beta}=\gamma(\alpha, \beta) u_{\alpha+\beta}$, where $\gamma(\alpha, \beta)$ is a multiplier for $\Gamma$, and $\alpha \rightarrow u_{\alpha}$ is a unitary multiplier 
representation with multiplier $\gamma . \Gamma$ is either a dense subgroup of $\mathbb{R}$ or discrete i.e. $\Gamma=\{n \alpha, n \in \mathbb{Z}\}$. If $\Gamma$ is discrete, then the strongly closed subalgebra $M_{\Gamma}$ generated by the root operators is abelian.

The restriction of $\Phi_{t}$ to $M_{\Gamma}$ is obviously an automorphism and as in the discrete case we get that the restriction of $\xi$ to $M_{\Gamma}$ is a trace. In the special case where $\Gamma$ is discrete, so that $\Gamma=\{n \alpha, n \in \mathbb{Z}\}, M_{\Gamma}$ is abelian and generated by the root operator $u$ corresponding to $\alpha$. Let $u$ be normalized to be unitary, then $M_{\Gamma}$ is simply the von Neumann algebra generated by $u$. Since $\Phi_{t}$ restricted to $M_{\Gamma}$ is a one parameter group of automorphism, it is induced by a one parameter flow on the spectrum of $u$. Since $\Phi_{t}\left(u^{n}\right)=e^{i t \alpha n} u^{n}$ this flow on the spectrum of $u$ must coincide with the flow $e^{i \varphi} \rightarrow e^{i(\varphi+\alpha t)}$ on the spectrum of $u$. From the fact that 1 is an eigenvalue of multiplicity one for the semigroup $\Phi_{t}$ restricted to $M_{\Gamma}$ it follows that $\left(M_{\Gamma}, \Phi_{t}, \xi\right)$ is an ergodic dynamical flow so that the flow $e^{i \varphi} \rightarrow e^{i(\varphi+\alpha t)}$ is ergodic with respect to the spectral measure $\mu$ for $u$ in $\xi$, i.e. the measure $\mu$ such that

$$
\xi(f(u))=\int_{|z|=1} f(z) d \mu(z) .
$$

Hence $\mu$ is an invariant and ergodic measure with respect to the flow induced by the rotation of the unit circle. Hence since $\Phi_{t}$ is also strongly continuous, we have that $d \mu$ is the Haar measure on the unit circle, and that $u$ has constant spectral multiplicity. We have thus the following theorem.

Theorem 2.8. Let $\left(M, \Phi_{t}, \xi\right)$ be an ergodic dynamical flow, and let $\Gamma$ be its root system. Then the restriction of $\xi$ to the von Neumann algebra $M_{\Gamma}$ generated by the root operators is a trace, $\Phi_{t}$ leaves $M_{\Gamma}$ invariant and the restriction of $\Phi_{t}$ to $M_{\Gamma}$ is a one parameter group of automorphisms. Moreover $\left(M_{\Gamma}, \Phi_{t}, \xi\right)$ is an ergodic dynamical flow. $\Gamma$ consists either of one point, or is discrete or is dense. In the discrete case we have $\Gamma=\{n \alpha ; n \in \mathbb{Z}\}$. Let in this case $u$ be a normalized root operator corresponding to $\alpha$. Then $u$ has Lebesgue spectrum and in fact the spectral measure for $u$ in the state $\xi$ is the Haar measure on the unit circle and $u$ has constant spectral multiplicity. Moreover the flow $\left(M_{\Gamma}, \Phi_{t}, \xi\right)$ is induced by rotating the spectrum of $u$ at the constant speed $\alpha$.

Remark. $t \rightarrow \Phi_{t}$ is an ergodic action of $\mathbb{R}$ on the von Neumann algebra $M_{\Gamma}$ with a discrete spectrum. In [27, Chapter 8$]$ we have an exhaustive discussion of such actions and we refer the interested reader to this reference for further information.

Acknowledgements. We would like to thank Professor E. St $\phi$ rmer and Dr. Chr. Skau for very instructive discussions. We are also very grateful to Professor $\mathrm{H}$. Araki for making a number of comments and suggestions which greatly improved the presentation of this paper. We thank Professor D. Evans for pointing out a gap in an earlier version. We also are grateful to Mrs. Mфller for her nice typing.

The first author is very grateful to the Institute of Mathematics, University of Oslo, for the warm hospitality. The financial support of the Norwegian Research Council for Science and the Humanities is also gratefully acknowledged.

\section{References}

1. a) Perron, O.: Grundlagen für eine Theorie der Jacobischen Kettenbruchalgorithmen. Math. Ann. 64, 1-76 (1907)

b) Perron, O. : Zur Theorie der Matrices. Math. Ann. 64, 248-263 (1908) 
2. a) Frobenius, F. G. : Über Matrizen aus positiven Elementen, I, II. Sitzungsber. Akad. Wiss. Berlin, Phys. Math. kl. 471-476 (1908); 514-518 (1909)

b) Frobenius, F. G. : Über Matrizen aus nicht negativen Elementen. Sitzungsber. Akad. Wiss. Berlin, Phys. Math. kl. 456-477 (1912)

3. Gantmacher, F. R. : Applications of the theory of matrices. New York: Interscience 1959

4. Evans, D., Hфegh-Krohn, R. : Spectral properties of positive maps on $\mathrm{C}^{*}$-algebras. J. London Math. Soc. 17, 345-355 (1978)

5. Krein, M. G., Rutman, M. A. : Linear operators leaving invariant a cone in a Banach space. Am. Math. Soc. Transl. 10, 199-235 (1950)

6. Jentzsch, R. : Über Integralgleichungen mit positivem Kern. J. reine angew. Math. 141, 235-244 (1912)

7. Karlin, S. : Positive operators. J. Math. Mech. 8, 907-937 (1959)

8. Rota, G. C. : On the eigenvalues of positive operators. Bull. Am. Math. Soc. 67, 556-558 (1961)

9. Schaefer, H. H. : Banach lattices and positive operators. Berlin, Heidelberg, New York: Springer 1974

10. Koopman, B. O. : Hamiltonian systems and transformations in Hilbert spaces. Proc. Nat. Acad. Sci. 17, 315-318 (1931)

11. Carleman, T. : Application de la théorie des équations intégrales singulières aux équations différentielles de la dynamique. Ark. Mat., Astr. Fys. 22B, No. 7 (1931)

12. von Neumann, J. : Zur Operatorenmethode in der klassischen Mechanik. Ann. Math. 33, 587 642 (1932)

13. Jacobs, K. : Lecture notes on ergodic theory. Aarhus University (1962/63)

14. a) St $\phi$ rmer, E. : Spectra of ergodic transformations. J. Funct. Anal. 15, 202-215 (1974)

b) St $\phi$ rmer, E. : Spectral subspaces of automorphisms. In: Rendic. S. I. F., Varenna, LX,

D. Kastler (ed.), pp. 128-138. New York: Academic Press 1976

15. Olesen, D. : On spectral subspaces and their applications to automorphism groups. In: Symposia mathematica XX, Ist. Naz. Alta Mat., pp. 253-296. London: Academic Press 1976

16. Kastler, D. : Equilibrium states of matter and operator algebras. In: Symposia mathematica XX, Ist. Naz. Alta Mat., pp. 49-107. London: Academic Press 1976

17. Evans, D., Sund, T. : Spectral subspaces for compact actions. Preprint, Oslo University (1977)

18. Kadison, R. : A generalized Schwarz inequality and algebraic invariants for operator algebras. Ann. Math. 56, 494-503 (1952)

19. Choi, M. D. : A Schwarz inequality for positive linear maps on $\mathrm{C}^{*}$-algebras. III. J. Math. 18, 565-574 (1974)

20. a) St $\phi$ rmer, E. : Positive linear maps on operator algebras. Acta Math. 110, 233-278 (1963)

b) St $\phi r m e r, E .:$ Positive linear maps of $\mathrm{C}^{*}$-algebras. In: Lecture notes in physics, Vol. 29, pp. 85-106. Berlin, Heidelberg, New York: Springer 1974

21. Evans, D. E. : Irreducible quantum dynamical semigroups. Preprint, Oslo University (1976)

22. Davies, E. D. : Quantum theory for open systems. New York: Academic Press 1976

23. a) Emch, G. G. : Non abelian specialK-flows. J. Funct. Anal. 19, 1-12 (1975)

b) Emch, G. G. : Generalized K-flows. Commun. math. Phys. 49, 191-215 (1976)

24. Albeverio, S., Hфegh-Krohn, R. : Dirichlet forms and Markov semigroups on $\mathrm{C}^{*}$-algebras. Commun. math. Phys. 56, 173-187 (1977)

25. Gorini, V., Frigerio, A., Verri, M., Kossakowski, A., Sudarshan, E. C. G. : Properties of markovian master equations. Preprint, University of Texas (1977)

26. Emch, G. G., Albeverio, S., Eckman, J. P. : Quasi free generalized K-flows. Rept. Math. Phys. 13, 73-85 (1978)

27. Albeverio, S., Hфegh-Krohn, R. : Ergodic actions of compact groups on von Neumann algebras. Preprint (Nov. 1977)

Communicated by $\mathrm{H}$. Araki

Received December 5, 1977; in revised form April 15, 1978 